\title{
Editorial
}

Bundesgesundheitsbl 2015 $\cdot 58: 921-922$

DOI 10.1007/s00103-015-2233-3

Online publiziert: 15. August 2015

๑) Springer-Verlag Berlin Heidelberg 2015
Susanne Jordan ${ }^{1}$ Jürgen Töppich ${ }^{2}$

${ }^{1}$ Robert Koch-Institut (RKI), Abteilung für Epidemiologie und Gesundheitsmonitoring, Berlin, Deutschland

2 Bundeszentrale für gesundheitliche Aufklärung (BZgA), Köln, Deutschland

\section{Die Förderung von Gesundheitskompetenz (Health Literacy) - Eine gesamtgesellschaftliche Aufgabe}

Die Fähigkeiten und Fertigkeiten, Gesundheitsinformationen $\mathrm{zu}$ finden, $\mathrm{zu}$ verstehen, zu bewerten und für gesundheitsbezogene Entscheidungen anzuwenden, werden als „Gesundheitskompetenz" bezeichnet, international wird von „Health Literacy“ gesprochen.

Je ausgeprägter die Gesundheitskompetenzen sind, so die allgemeine Annahme, desto besser ist man in der Lage, sich im Alltag über das Gesundheitswesen, die Prävention von Krankheiten und die Gesundheitsförderung zu informieren, eine Meinung zu bilden und Entscheidungen zu treffen, die die Lebensqualität und Gesundheit im Lebensverlauf erhalten oder verbessern. Das Ausmaß von Gesundheitskompetenz in der Bevölkerung ist jedoch nicht nur von individuellen Voraussetzungen und erworbenen Kompetenzen abhängig, sondern ganz wesentlich auch von der fachlichen Qualität, Angemessenheit, Verständlichkeit, Sichtbarkeit, Verfügbarkeit und Vermittlungsform der bereitgestellten Informationen. Damit rücken auch Anbieter und Leistungserbringer von Gesundheitsinformationen in den Blick, wenn es um die Förderung von Gesundheitskompetenzen geht. Gesundheitskompetenz ist somit das Ergebnis gelungener Aneignung auf der Basis individueller und systembedingter Leistungen.

Dem Thema „Gesundheitskompetenz" wird international schon seit längerem hohe Aufmerksamkeit geschenkt und auch in Deutschland findet dieses umfassende Konzept von Gesundheitskompetenz/Health Literacy in der Krankenversorgung sowie der Prävention und Ge- sundheitsförderung zunehmend Beachtung.

Ziel dieses Schwerpunktheftes ist es, einen Überblick über den Stand der Diskussion des Konzeptes Gesundheitskompetenz (Health Literacy) primär im deutschsprachigen Raum zu geben und dessen Anwendung in den Feldern Forschung, Prävention und Krankenversorgung exemplarisch in vier thematischen Blöcken vorzustellen. Die beiden Beiträge des ersten Themenblocks zu Konzepten der Gesundheitskompetenz befassen sich mit der Operationalisierung des Konzeptes sowie der Generalisierbarkeit für alle Bevölkerungsgruppen. Es folgen zwei Beiträge zum aktuellen Wissensstand über die Verbreitung der Gesundheitskompetenz in Deutschland. Im dritten Abschnitt des Schwerpunktheftes wird die Förderung von Gesundheitskompetenz im Handlungsfeld Prävention und Gesundheitsförderung exemplarisch für bestimmte Bevölkerungsgruppen und Settings diskutiert. Abschließend zeigen zwei Beiträge die Bedeutung von Gesundheitskompetenz in der Krankenversorgung und welche Möglichkeiten der Förderung von Gesundheitskompetenz in diesem Kontext denkbar sind. Diese Auswahl versucht den aktuellen Stand in Forschung und Praxis zur Gesundheitskompetenz/Health Literacy in Deutschland abzubilden.

Im ersten Beitrag des Schwerpunktheftes untersuchen T. Abel und K. Sommerhalder Konzept und Operationalisierung von Gesundheitskompetenz/Health Literacy und geben einen Überblick über aktuelle Definitionen von Gesundheitskom- petenz. Sie dokumentieren, dass Gesundheitskompetenz heute vielfach definiert und operationalisiert wird und skizzieren Stärken und Schwächen der derzeitigen Ansätze zur Konzipierung wie auch zur Messung von Gesundheitskompetenz. O. Okan, P. Pinheiro, P. Zamora und U. Bauer fokussieren in ihrem Beitrag das Thema „Health Literacy von Kindern und Jugendlichen" mit dem Ziel, den aktuellen Forschungsstand sowie einige der Desiderata in der Diskussion über Health Literacy im Kindes- und Jugendalter zu beschreiben. Sie stellen das Fehlen konzeptioneller Zugänge hinsichtlich alters- und entwicklungsstufenangepasster Modelle fest. Dies erschwert den theoretischen und methodischen Zugang zum Thema „Health Literacy im Kindes- und Jugendalter". Hier erkennen sie einen dringenden Forschungsbedarf.

Teil zwei des Schwerpunktheftes beginnt mit einer empirischen Untersuchung zur Verteilung der Gesundheitskompetenz von Erwachsenen in Deutschland. S. Jordan und J. Hoebel nutzen dafür die Ergebnisse der Studie „Gesundheit in Deutschland aktuell“ (GEDA). Gemessen wird die Gesundheitskompetenz mit der Kurzform des European Health Literacy Questionnaire (HLS-EU-Q16), zusammen mit Fragen zur Soziodemografie, zum Gesundheitsverhalten und -zustand. Die Ergebnisse zeigen, dass ein erheblicher Teil der in Deutschland lebenden Erwachsenen nach eigener Einschätzung Schwierigkeiten beim Zugang zu sowie beim Verstehen, Bewerten und Anwenden von Gesundheitsinformationen hat und signalisieren damit einen deutli- 
chen Handlungsbedarf für größere Bevölkerungsgruppen. Dies insbesondere auch deshalb, weil ein niedriges Gesundheitskompetenzniveau mit einer schlechteren körperlichen sowie psychischen Gesundheit einhergeht. Die Einflussfaktoren und Folgen der Gesundheitskompetenz bildungsferner Jugendlicher stehen im Mittelpunkt der Untersuchung von G. Quenzel, D. Schaeffer, M. Messer und D. Vogt. Datengrundlage ist eine an den EuropeanHealth-Literacy-Survey anschließende vertiefende Erhebung in Nordrhein-Westfalen. Bildungsferne Jugendliche haben in dieser Studie im Durchschnitt eine deutlich niedrigere Gesundheitskompetenz als die Allgemeinbevölkerung. Das Risiko für eine niedrige Gesundheitskompetenz steigt noch einmal deutlich bei vorliegendem Migrationshintergrund. Die Ergebnisse zum Zusammenhang zwischen der Gesundheitskompetenz und gesundheitsbezogenen Verhaltensweisen sind jedoch uneinheitlich.

Zu Beginn des dritten Abschnitts zur Prävention und Gesundheitsförderung stellen T. Rohde, K. Kolpatzik und A.F. Winter in ihrem Beitrag über die Förderung der Gesundheitskompetenz älterer Menschen das EU-Projekt „Intervention Research On Health Literacy among Ageing population" (IROHLA) vor. An dem dreijährigen Projekt beteiligten sich 22 Partner aus neun europäischen Ländern, davon vier aus Deutschland. Das Projekt zeigt, dass es für die Förderung der Gesundheitskompetenz älterer Menschen umfassende Lösungsansätze braucht, in denen Maßnahmen für das Individuum, seine Umgebung, relevante Fachkräfte und Strukturen im Gesundheitswesen aufeinander abgestimmt sind. T. Quast und G. Nöcker befassen sich in ihrem Beitrag mit einer innovativen Interventionsmethode zur Förderung von Gesundheitskompetenz. Die „virtuelle Streetwork“ in unterschiedlichen Internet-Foren zu Gesundheitsthemen für Schwangere und Jugendliche wurde von den Zielgruppen positiv aufgenommen. Zugleich bietet sie Forenbetreibern die Chance, von Forennutzenden mehr über die Nutzenden zu lernen und dadurch ihre institutionelle Gesundheitskompetenz zu erhöhen. Die Verbesserung der Gesundheitskompetenz durch informelles Lernen im Alltag der Arbeitswelt, insbesondere in kleinen und mittleren Unternehmen (KMU), steht im Zentrum des Beitrags von C. Eickholdt, W. Hamacher und N. Lenartz zur Förderung von Gesundheitskompetenz im Betrieb. Dabei bedarf es eines umfassenden Verständnisses von Gesundheitskompetenz, das an den Rahmenbedingungen der Arbeitswelt ansetzt.

Im letzten Abschnitt des Themenheftes zur Relevanz von Gesundheitskompetenz in der Krankenversorgung zeigen $E$. M. Bitzer und U. Spörhase in ihrem Beitrag die Bedeutung der Gesundheitskompetenz in der medizinischen Rehabilitation. Für sie bedeutet die Förderung der Gesundheitskompetenz zum einen Patientenschulungen an der empirischen Bildungsforschung kompetenzorientiert auszurichten und zugleich Rahmenbedingungen für formelles und informelles Lernen zu gestalten. Die Bedeutung von Organisationen für die Entwicklung von individueller Gesundheitskompetenz veranschaulichen J. M. Pelikan und C. Dietscher in ihrem Beitrag über gesundheitskompetente Krankenhäuser. Die organisationale Gesundheitskompetenz von Krankenhäusern zeigt sich in einer Sensibilität für das Thema "Gesundheitskompetenz" innerhalb der Einrichtung. Dies wird am Beispiel des Wiener Konzeptes „Gesundheitskompetenter Krankenbehandlungsorganisationen " erläutert, das beispielsweise das Management ebenso einbezieht wie Mitarbeiter/-innen oder Navigationshilfen für Patientinnen und Patienten sowie Besucher/-innen.

Erkennbar wird, dass das komplexe Thema "Gesundheitskompetenz" derzeit in Deutschland verstärkt in den Blick genommen wird. Unterschiedliche Disziplinen und Akteure sind mit relevanten Aspekten, Fragestellungen und Ansätzen befasst. Die Beiträge zeigen, dass sich die Diskussion im Stadium des Aufbaus und der Weiterentwicklung befindet. Dies gilt sowohl für die theoretischen Grundlagen, die theoriebasierte Erarbeitung von Erhebungsinstrumenten als auch für die Interventionspraxis. Die ersten empirischen Ergebnisse zur Verteilung der Gesundheitskompetenz in Deutschland liegen nun vor. Die teilweise uneinheitlichen Befunde zeigen hier weiteren Forschungs- bedarf an. Deutlich wird auch, dass besonders Maßnahmen für Bevölkerungsgruppen mit niedriger Gesundheitskompetenz benötigt werden. Insgesamt weist dies auf die Erfordernis einer verstärkten Alters- und Zielgruppenspezifität in der Theorie- und Interventionsentwicklung zur Gesundheitskompetenz hin. Eine besondere Rolle bei der Förderung von Gesundheitskompetenz kommt hierbei Ansätzen zu, die die Sensibilität von Organisationen für Gesundheitskompetenz als notwendige Komponente in die Förderung individueller Gesundheitskompetenz einbezieht. Eine zukünftige Strategie - so vielfältige Hinweise - hätte die verschiedenen gesellschaftlichen Ebenen einzubinden, um die Gesundheitskompetenz von Einzelnen und Organisationen zu stärken.

Wir wünschen den Leserinnen und Lesern eine spannende Lektüre und wünschen uns, dass von diesem Themenheft konstruktive Impulse für die Auseinandersetzung mit dem Thema „Gesundheitskompetenz" ausgehen.

Ihre<smiles>CCOC(C)C(C)O</smiles>

Susanne Jordan

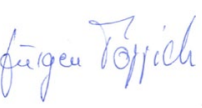

Jürgen Töppich

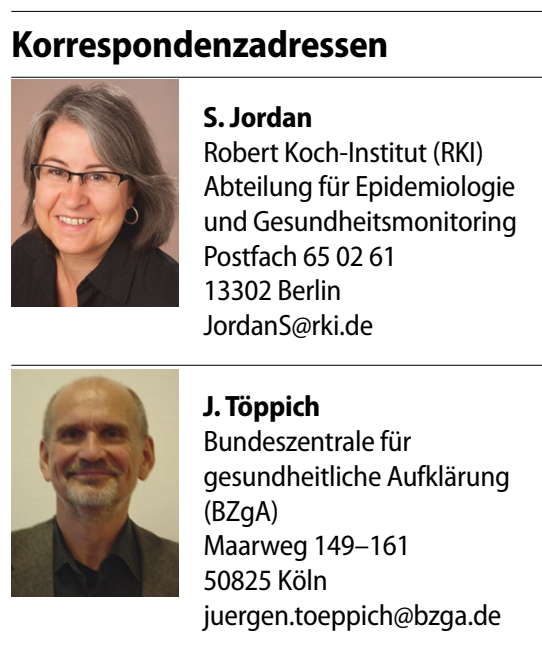

